\title{
Accelerating FPGA Routing Using Architecture-Adaptive A* Techniques
}

\author{
Akshay Sharma \\ Actel Corporation \\ Mountain View, CA - 94043, USA \\ Akshay.Sharma@actel.com
}

\author{
Scott Hauck \\ University of Washington \\ Seattle, WA - 98195, USA \\ hauck@ee.washington.edu
}

\begin{abstract}
The $A^{*}$ algorithm is a well-known path-finding technique that is used to speed up FPGA routing. Previously published $A^{*}$-based techniques are either targeted to a class of architecturally similar devices, or require prohibitive amounts of memory to preserve architecture adaptability. This work presents architecture-adaptive $A^{*}$ techniques that require significantly less memory than previously published work. Our techniques are able to produce routing runtimes that are within 7\% (on an islandstyle architecture) and 9\% better (on a hierarchical architecture) than targeted heuristic techniques. Memory improvements range between $30 X$ (islandstyle) and 140X (hierarchical architecture).
\end{abstract}

\section{Introduction}

Routing is an important step in the FPGA toolflow. FPGAs have a finite number of discrete routing resources, and the effectiveness of an FPGA router directly impacts the performance of an application netlist on a target device. Pathfinder [8] is the current, state-of-the-art FPGA routing algorithm. Pathfinder uses an iterative, negotiation-based approach to solve the FPGA routing problem. During the first routing iteration, nets are freely routed without paying attention to resource sharing. Individual nets are routed using a shortest path graph algorithm. At the end of the first iteration, resources are generally congested because multiple nets have shared them. During subsequent iterations, the cost of using a resource is increased based on the number of nets that share the resource, and the history of congestion on that resource. In effect, nets are made to negotiate for routing resources. If a resource is highly congested, nets that can use lower congestion alternatives are forced to do so. On the other hand, if the alternatives are more congested than the resource, then a net may still use that resource.

Pathfinder has proved to be one of the most powerful FPGA routing algorithms to date. Pathfinder's negotiation-based framework is a very effective technique for routing nets on FPGAs. More importantly, Pathfinder is a truly architectureadaptive routing algorithm. The algorithm operates on a directed graph abstraction of an FPGA's interconnect structure, and can thus be used to route netlists on any FPGA that can be represented as a directed routing graph. We believe that Pathfinder's adaptability is one of the main reasons for its widespread acceptance.

When routing an individual net, Pathfinder uses a greedy search algorithm that is similar to Dijkstra's algorithm [5]. A net that has $n$ sink terminals is routed using $n$ searches. Further, each net may be ripped up and rerouted multiple times as the algorithm progresses through routing iterations. All in all, employing a search-based algorithm to do FPGA routing is a computationally expensive process.

A path-finding technique that is commonly used to speed up graph-based search is the A* algorithm [9]. The $A^{*}$ algorithm speeds up routing by pruning the search space of Dijkstra's algorithm. The search space is pruned by preferentially expanding the search wavefront in the direction of the target node. When the search is expanded around a given wire, the routing algorithm expands the search through the neighbor wire that is nearest the target node. This form of directed search is accomplished by augmenting the cost of a routing wire with a heuristically calculated estimate of the cost to the target node.

$$
\text { Equation 1: } f_{n}=g_{n}+h_{n}
$$

Consider Equation 1, in which $g_{n}$ is the cost of a shortest path from the source to wire $n$, and $h_{n}$ is a heuristically calculated estimate of the cost of a shortest path from $n$ to the target node (hereafter, we refer to this estimate as a 'cost-to-target' estimate). The value $f_{n}$ is the estimated cost of a shortest path from the source to the target that contains the wire $n$. The $\mathrm{A}^{*}$ algorithm uses $f_{n}$ to determine the cost of expanding the search through wire $n$. Note that Dijkstra's algorithm uses only $g_{n}$ to calculate the cost of wire $n$. 
To guarantee optimality, the cost-to-target estimate $h_{n}$ at a given wire $n$ must be less than or equal to the actual cost of the shortest path to the target. Overestimating the cost to the target node may provide even greater speedups, but then the search is not guaranteed to find an optimal path to the target. Currently, there is no architecture-adaptive, memory efficient technique for performing $\mathrm{A}^{*}$ search on FPGAs. Our goal in this paper is the development of architecture-adaptive $A^{*}$ techniques that can be used to speed up the FPGA routing process.

\section{Previous work}

The work described in $[11,12]$ discusses directed search techniques that can speed up the Pathfinder algorithm. These techniques are similar to the $\mathrm{A}^{*}$ algorithm, and use a formulation like the one shown in Equation 1 to calculate the cost of expanding the search through a routing wire. During the routing process, cost-to-target estimates are heuristically calculated using geometric information. Estimate calculations often require potentially complex operations, and the cost of calculating estimates can slow down the router. Further, the techniques presented in $[11,12]$ may need to be re-implemented whenever the interconnect architecture is changed, and are not suitable for non-Manhattan interconnect structures. Two examples of such interconnect structures are shown in Figure 1. The architecture on the left [2] provides different types of routing resources in the horizontal and vertical directions, and the architecture on the right [6] has a strictly hierarchical interconnect structure.
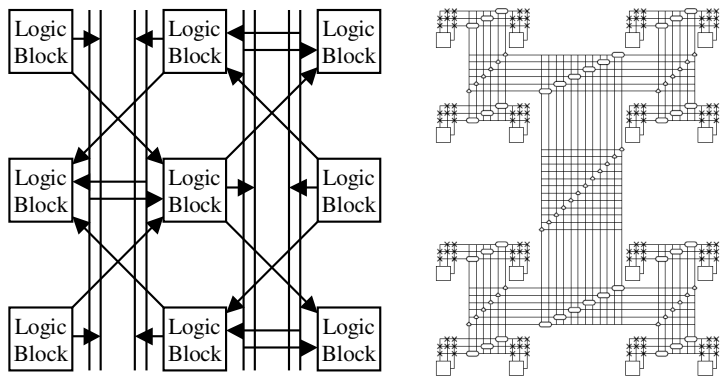

Figure 1. Non island-style interconnect structures $[2,6]$.

Recall that Pathfinder's primary strength is its adaptability to different FPGA architectures. Existing A* techniques violate this assumption, since they hard-code interconnect assumptions into the cost-totarget estimators. In this paper, we present architecture-adaptive runtime enhancements to the Pathfinder algorithm. Our techniques are also based on using the $\mathrm{A}^{*}$ algorithm to speed up the search process. However, our methodology is adaptive and does not rely on architecture-specific heuristic costto-target estimates. The techniques presented in this paper are routability-driven. Extending these techniques to include timing information may be accomplished in a manner similar to that described in [1].

\section{Architecture-adaptive A* techniques}

The developers of the Pathfinder algorithm briefly discussed the idea of using the $\mathrm{A}^{*}$ algorithm to speed up routing [8]. They proposed the use of a precomputed lookup table that would hold the cost of a shortest path from every routing wire to every sink terminal in the interconnect structure. Specifically, there would be a separate entry for every routing wire in this lookup table, and each entry would hold costto-target estimates for all sink terminals in the interconnect structure. During routing, the cost-totarget estimate at a routing wire could then be obtained using a simple table lookup.

Pre-computing and tabulating cost-to-target estimates in this fashion is indeed an adaptive scheme. Shortest paths can be calculated using Dijkstra's algorithm, and no architecture-specific information is required. The approach also guarantees an exact estimate of the shortest path in the absence of routing congestion. However, while the computational complexity of this approach is manageable, the space requirements for routing-rich structures may explode. Assuming an island-style, 10-track, 100x100 FPGA that has only single-length segments, the memory required to store the cost-to-target lookup table would be measured in GigaBytes. Memory requirements of this size are probably impractical.

Sharing a table entry among multiple routing wires that have similar cost-to-target estimates can reduce the memory requirement of the lookup table. For example, if one hundred wires share each table entry, the size of the table may be reduced by one hundred times. The cost-to-target estimate for a given sink terminal is the same for all wires that share the table entry, and can be calculated using a Dijkstra search that begins at the wire closest to the target. Specifically, the entire set of wires that share a table entry constitutes a "super" source node for the Dijkstra search. In this manner, we ensure that the cost-to-target estimate for a given sink terminal is the cost of a shortest path from the wire that is closest to the sink terminal. From this point on, we will refer to this method for calculating cost-to-target estimates as the superDijkstra method.

The important question now is how to identify wires that should share a table entry. Clearly, we would like to identify clusters of wires that have similar cost-to-target estimates, so that we can collect them together in a set that points to a single entry in the cost-to-target lookup table. Our first technique for clustering wires together is inspired by two observations: 
- The number of logic units in an FPGA is generally much less than the number of interconnect wires.

- Logic units and interconnect wires are often interspersed in the FPGA fabric in a regular fashion.

Based on these observations, our first technique uses a proximity metric (described in the Section 4) to associate each wire with a logic unit. After each interconnect wire has been associated with a logic unit, all wires associated with the same logic unit are assigned to the same cluster. The cost-to-target estimates for each cluster are calculated using the superDijkstra method and stored in a lookup table. Since the number of table entries is equal to the number of logic units, the memory requirements of this technique are significantly less than a lookup table that has a separate entry for each wire in the interconnect structure.

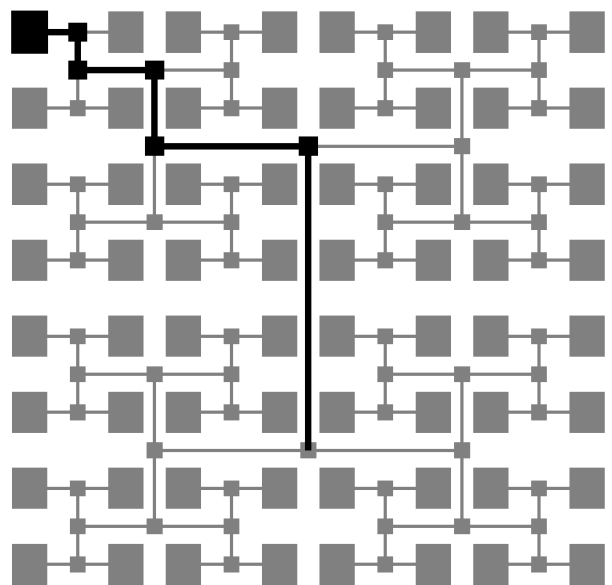

Figure 2: An example of a tree-based, hierarchical interconnect structure. Assume that the wires shown in black belong to the same cluster.

The associate-with-closest-logic-unit technique is probably well suited to island-style FPGAs. Since the logic and interconnect structures of an island-style FPGA are closely coupled, this approach may produce clusters of wires that have reasonably similar cost-to-target estimates. On hierarchical structures, the accuracy of an associate-with-closest-logic-unit approach may not be quite as good. For example, consider the tree-like interconnect structure in Figure 2. The routing wire that is topmost in the interconnect hierarchy is equally close to all logic units, while the wires in the next level are equally close to half the logic units, and so on. Associating wires with individual logic units in a strictly hierarchical interconnect structure may result in large cost-to-target underestimates.

In Figure 2, assume that the wires shown in black are associated with the black logic unit, and that the cost-to-target estimates for the cluster have been calculated using the superDijkstra method. The wire that directly connects to the black logic unit will have a cost-to-target estimate of five for the logic units in the northeast, southeast and southwest quadrants of the architecture. Note that the actual cost is nine wires for the northeast quadrant, and ten for the southeast and southwest quadrants. Estimates that are a factor of two below exact might slow down the router considerably. However, every wire in the cluster shown in Figure 2 does not suffer from the same problem. The cluster wire that is topmost in the interconnect hierarchy (black vertical line down the middle of Figure 2) will have exact cost-to-target estimates for all logic units in the northeast, southeast and southwest quadrants, and underestimates for logic units in the northwest quadrant.

To summarize, one would expect the associatewith-closest-logic-unit approach to work well for island-style structures. However, due to the approach's potential limitations on hierarchical structures, we feel that a more sophisticated technique might be necessary to produce reasonably accurate cost-to-target estimates across different interconnect styles.

\section{K-means clustering}

Our second technique for architecture adaptive clustering problem is to use the K-means algorithm, guided by each resource's cost-to-target estimates. Kmeans clustering is an iterative heuristic that is used to divide a dataset into $\mathrm{K}$ non-overlapping clusters based on a proximity metric. Pseudocode for the KMeans algorithm appears in Figure 3.

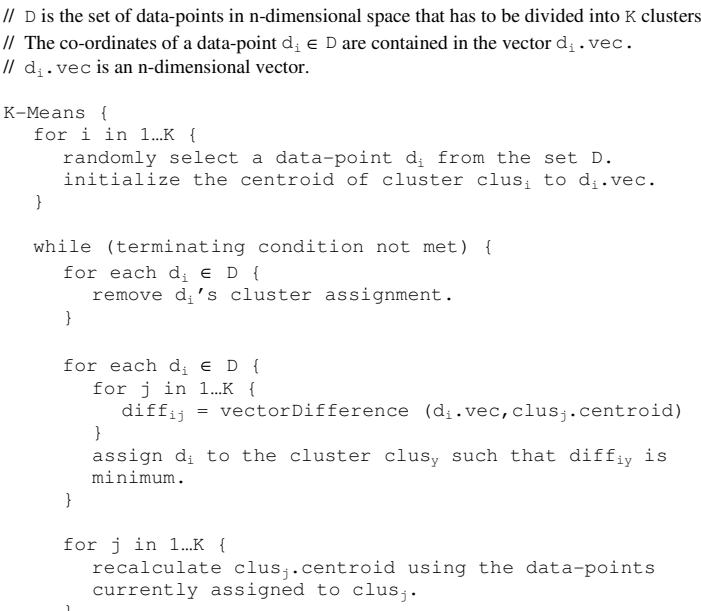

Figure 3: Pseudocode for the K-Means clustering algorithm. 
We now briefly describe our choices for the alparameters that characterize the K-Means algorithm.

Dataset (D): The dataset D simply consists of all the routing wires in the interconnect structure of the target device.

Number of Clusters (K): We experimentally determined that a value of $\mathrm{K}$ greater than or equal to the number of logic units in the target device is a reasonable choice. Section 5 describes the effect of $\mathrm{K}$ on the quality of clustering solutions.

Initial Seed Selection: The initial seeds consist of $\mathrm{K} / 2$ randomly selected logic-block output wires and $\mathrm{K} / 2$ randomly selected routing wires.

Terminating Condition: The K-Means algorithm is terminated when less than $1 \%$ of the dataset changed clusters during the previous clustering iteration.

Calculating Cost-to-Target Estimates: On completion of the clustering algorithm, the actual $\mathrm{A}^{*}$ estimates for a cluster are calculated using the superDijkstra method.

Co-ordinate Space and Proximity Metric: The most important consideration in applying the $\mathrm{K}$ Means algorithm to solve the interconnect clustering problem is the proximity metric. Specifically, we need to determine a co-ordinate space that is representative of the $\mathrm{A}^{*}$ cost-to-target estimate at each wire in the dataset. In our implementation, the co-ordinates of a routing wire represent the cost of the shortest path to a randomly chosen subset $\mathrm{S}$ of the sink terminals in the interconnect structure. The coordinates of each routing wire are pre-calculated using Dijkstra's algorithm and stored in a table.

If the number of sink terminals in $\mathrm{S}$ is $n$, then the co-ordinates of a routing wire $d_{i} \in \mathrm{D}$ are represented by an n-dimensional vector $d_{i}$.vec. Each entry $c_{i j}(j \in$ $1 . . \mathrm{n})$ in the vector $d_{i} . v e c$ is the cost of a shortest path from the routing wire $d_{i}$ to the sink terminal $j$. The co-ordinates for all $d_{i} \in \mathrm{D}$ are calculated by launching individual Dijkstra searches from each sink terminal in the set $\mathrm{S}$. Note that the edges in the underlying routing graph are reversed to enable Dijkstra searches that originate at sink terminals. At the end of a Dijkstra search that is launched at sink terminal $j$, the cost of a shortest path from every $d_{i}$ to the terminal $j$ is written into the corresponding $c_{i j}$ entry of divec. The vector $d_{i}$.vec is used by the $\mathrm{K}$ Means algorithm to calculate the "distance" between the wire $d_{i}$ and the centroid of each cluster. The distance between $d_{i}$ and a cluster centroid is defined as the magnitude of the vector difference between $d_{i}$ vec and the cluster centroid.

Note that the size of $\mathrm{S}$ directly influences the memory requirements of our clustering implementation. In the extreme case where $\mathrm{S}$ contains every sink terminal in the target device, the memory requirements would match the prohibitively large requirements of a table that stores the cost of a shortest path from each routing wire to every sink terminal. This would undermine the purpose of using a clustering algorithm to reduce the memory requirements of an $\mathrm{A}^{*}$ estimate table. It is thus useful to sub-sample the number of sink terminals in the target device when setting up the set $\mathrm{S}$.

\begin{tabular}{|c|c|c|c|c|}
\hline & & Pathfinder & Cluste & ring \\
\hline Size & ChanWidth & $|\mathbf{S}|=\mathbf{N}_{\mathrm{T}}$ & $|\mathrm{S}|=0.06^{\star} \mathrm{N}_{\mathrm{T}}$ & Estimates \\
\hline $10 \times 10$ & 10 & 0.0012 & 0.0001 & 0.0001 \\
\hline $20 \times 20$ & 10 & 0.0151 & 0.0009 & 0.0007 \\
\hline $30 \times 30$ & 10 & 0.0707 & 0.0043 & 0.0035 \\
\hline $40 \times 40$ & 10 & 0.2152 & 0.0130 & 0.0106 \\
\hline $50 \times 50$ & 10 & 0.5132 & 0.0310 & 0.0253 \\
\hline $60 \times 60$ & 10 & 1.0474 & 0.0631 & 0.0518 \\
\hline $70 \times 70$ & 10 & 1.9185 & 0.1155 & 0.0949 \\
\hline $80 \times 80$ & 10 & 3.2449 & 0.1951 & 0.1607 \\
\hline $90 \times 90$ & 10 & 5.1629 & 0.3103 & 0.2559 \\
\hline $100 \times 100$ & 10 & 7.8268 & 0.4703 & 0.3882 \\
\hline $110 \times 110$ & 10 & 11.4087 & 0.6854 & 0.5662 \\
\hline $120 \times 120$ & 10 & 16.0986 & 0.9669 & 0.7994 \\
\hline $130 \times 130$ & 10 & 22.1044 & 1.3275 & 1.0980 \\
\hline $140 \times 140$ & 10 & 29.6517 & 1.7805 & 1.4735 \\
\hline $150 \times 150$ & 10 & 38.9842 & 2.3406 & 1.9380 \\
\hline $160 \times 160$ & 10 & 50.3636 & 3.0236 & 2.5045 \\
\hline $170 \times 170$ & 10 & 64.0690 & 3.8462 & 3.1869 \\
\hline $180 \times 180$ & 10 & 80.3979 & 4.8262 & 4.0001 \\
\hline $190 \times 190$ & 10 & 99.6654 & 5.9825 & 4.9599 \\
\hline $200 \times 200$ & 10 & 122.2044 & 7.3351 & 6.0828 \\
\hline
\end{tabular}

Table 1 compares the memory requirements of a clustering-based implementation that sub-samples the sink terminals with a table that stores the cost of a shortest path from each routing wire to every sink terminal in the target device. The target architecture is assumed to be a square island-style array that has only single-length wire segments. In our calculations, we assume that the sizes of a floating-point number, integer number, and a pointer are all four bytes. Column 1 lists the size of the target array, and column 2 lists the channel width of the target array. Let the total number of sink terminals in the target array be $N_{T}$. Column 3 lists the memory requirements of a table that stores the cost of a shortest path from each wire to every sink terminal in the target device (i.e. $|\mathrm{S}|=N_{T}$ ). This corresponds to the exhaustive lookup table approach proposed by the creators of the Pathfinder algorithm in [8]. Column 4 lists the size of a table that stores costs to only $6 \%$ of the sink terminals $\left(|\mathrm{S}|=0.06^{*} N_{T}\right)$, and column 5 lists the size of a table that holds cost-to-target estimates for the clusters produced by a K-Means implementation where $\mathrm{K}=$ number of logic units in the target device. All memory requirements are reported in Gigabyte. It is clear from Table 1 that our K-Means clustering approach avoids the impractical memory requirements of a table that stores costs to every sink terminal in the target device. 
Finally, note that the clustering process is a onetime preprocessing step that needs to be performed only on a per-architecture basis. The table of cost-totarget estimates produced by the clustering algorithm can be reused every time a new netlist is routed, and there is no additional runtime or memory cost incurred by our techniques on a per-netlist basis.

\section{Results}

We conduct three experiments to test the validity of using the K-Means algorithm to cluster the interconnect structure of an FPGA. The first experiment studies the effect of sub-sampling the sink terminals in the target device on the quality of clustering solutions. The second experiment studies the effect of the number of clusters $(\mathrm{K})$ on quality, and the third experiment compares the quality of clustering-based $\mathrm{A}^{*}$ estimates with heuristically calculated estimates. To evaluate the adaptability of our techniques, we conduct the experiments on an island-style interconnect architecture and HSRA [6]. Details of the architectural parameters used in our experiments can be found in [10].

Since the truest measure of the quality of an $\mathrm{A}^{*}$ estimate is routing runtime, our quality metric is defined to be the CPU runtime per routing iteration when routing a placement on the target device. The placements for our experiments on island-style structures are obtained using VPR [1], and the placements for our experiments on HSRA are produced using Independence [10].

Finally, note that our clustering techniques are guaranteed to produce conservative cost-to-target estimates, and hence these techniques have no effect on routing quality.

\subsection{Experiment 1 - Sub-sampling Sinks}

Experiment 1 studies the effect of sub-sampling the number of sink terminals in the target device. The set of benchmark netlists used in this experiment is a subset of the netlists shown in Table 2 (island-style) and Table 3 (HSRA).

Figure 4 shows the variation in quality of clustering solutions. The $\mathrm{x}$-axis represents the fraction of sink terminals that are used to represent the co-ordinates of each wire during clustering. The subset of sink terminals used in the experiment is randomly generated. The $y$-axis represents routing runtime measured in seconds per routing iteration. The curves show the variation in routing runtimes when using $\mathrm{A}^{*}$ estimates produced by the K-Means clustering technique. The flat line shows the routing runtime when using architecture-specific heuristic $A^{*}$ estimates. The value of $\mathrm{K}$ in this experiment is equal to the number of logic units in the target device.
Figure 4 shows that using as little as $5 \%$ of the sink terminals during clustering may be sufficient to produce estimates that are comparable to heuristic estimates. This is not a surprising result. Due to the regularity of an FPGA's interconnect structure, a small subset of sink terminals may be sufficient in resolving the interconnect wires into reasonably formed clusters. Note that $5 \%$ of the sink terminals represents a variable number of sink terminals across the set of benchmark netlists. Depending on the size of the netlist, $5 \%$ of the sink terminals could be anywhere between two and fifty sink terminals.
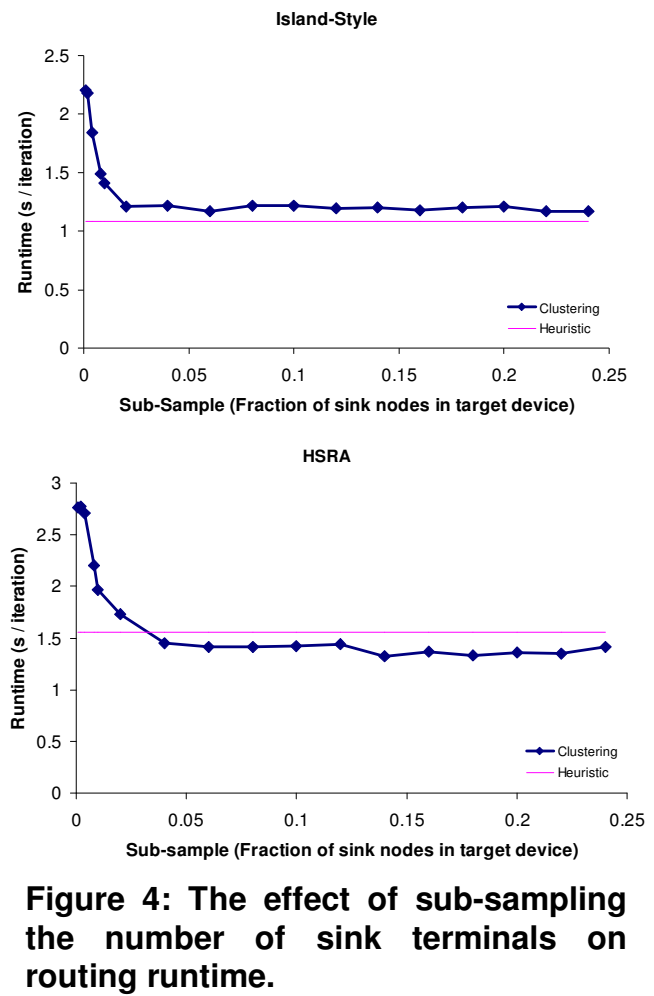

In Figure 5, we present the results of a second study that evaluates the quality of clustering solutions when using a small, fixed number of sink terminals. Figure 5 shows that using a small number (say 16) of randomly selected sink nodes may be enough to produce clustering solutions that are within approximately $15 \%$ of heuristic estimates.

\subsection{Experiment 2 - Number of Clusters (K)}

Experiment 2 studies the effect of the number of clusters $(\mathrm{K})$ on the quality of clustering solutions. The set of benchmark netlists used in this experiment is identical to the set used in Experiment 1. We use a sub-sample of $6 \%$ for island-style architectures, and $14 \%$ for HSRA. 

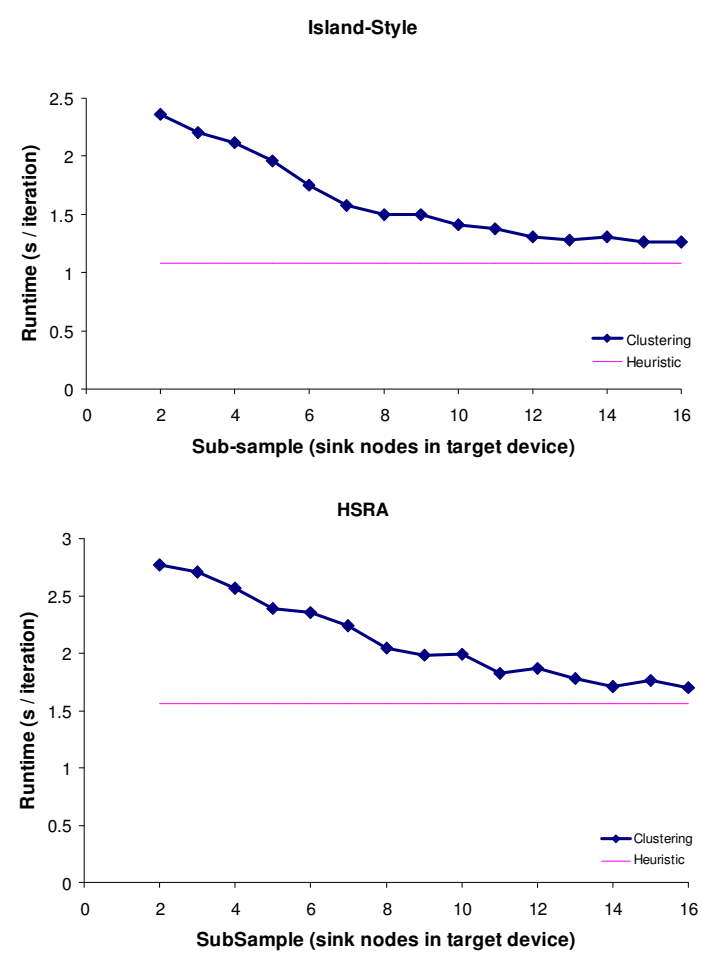

Figure 5: Using a small number of sink nodes may produce clustering solutions of acceptable quality.
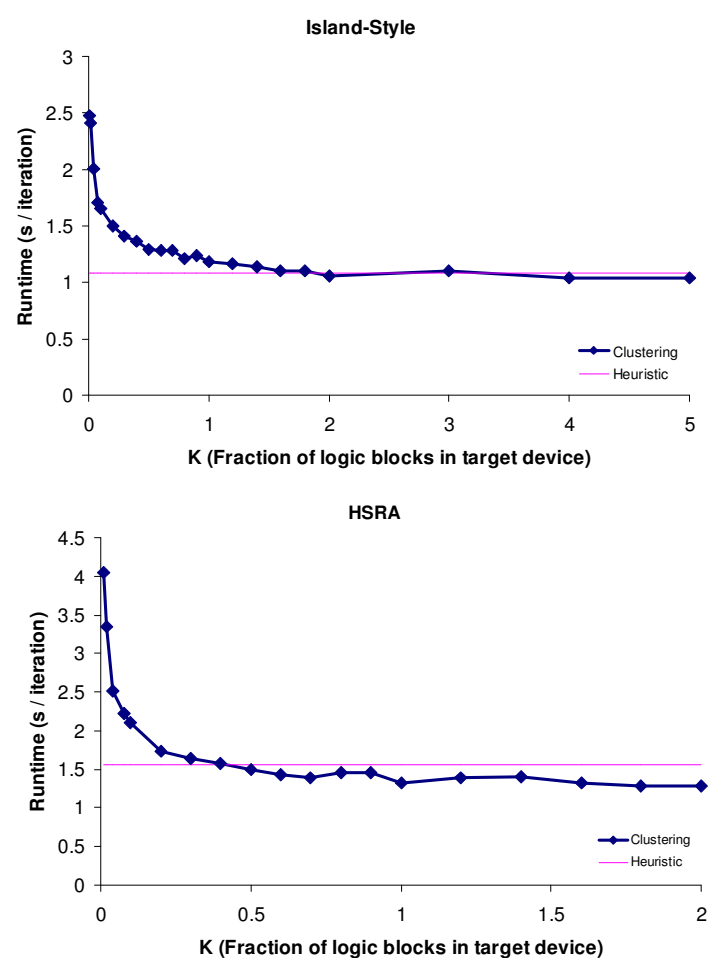

Figure 6: The effect of $K$ on routing runtime.
Figure 6 shows the effect of $\mathrm{K}$ on routing runtime. The $\mathrm{x}$-axis shows the value of $\mathrm{K}$ as a fraction of the number of logic units in the target device, and the $y$-axis shows routing runtime in seconds per routing iteration. The charts in Figure 6 show that a value of $\mathrm{K}$ equal to or greater than the number of logic units in the target device produces clustering solutions of qualities similar (within 10\%) to heuristic estimates.

\subsection{Quantitative Comparisons}

Experiment 3 is a quantitative comparison of the quality of the $\mathrm{A}^{*}$ estimates produced by our clustering techniques vs. heuristically calculated estimates. We use the following settings in this experiment:

- Associate-with-closest-logic-unit technique. This technique is implemented by running only the first iteration of K-Means clustering. $\mathrm{K}$ is chosen to be equal to the number of logic units in the target device $\left(\mathrm{K}=\mathrm{N}_{\mathrm{L}}\right)$, and initial seeds chosen to be logic unit outputs. The value of sink sub-sample is $6 \%\left(|\mathrm{~S}|=0.06 * \mathrm{~N}_{\mathrm{T}}\right)$. These settings represent a relatively low-effort clustering step. This step might be undertaken when clustering runtime and memory requirements need to be very low.

- K-Means clustering, with a sink sub-sample value of $6 \%\left(|\mathrm{~S}|=0.06^{*} \mathrm{~N}_{\mathrm{T}}\right)$ and $\mathrm{K}$ equal to the number of logic units in the target device $\left(K=N_{L}\right) . N_{T}$ is the total number of sink terminals in the target device, and $\mathrm{N}_{\mathrm{L}}$ is the total number of logic units in the target device. These settings represent an empirically determined sweet-spot for our KMeans clustering technique.

- K-Means clustering, with a sink sub-sample value of $20 \%\left(|\mathrm{~S}|=0.2 * \mathrm{~N}_{\mathrm{T}}\right)$ and $\mathrm{K}$ equal to twice the number of logic units in the target device $(\mathrm{K}=$ $2 * \mathrm{~N}_{\mathrm{L}}$ ). These are aggressive settings that represent potentially high quality clustering solutions. Such settings may be used when absolutely the best quality clustering solutions are required, and clustering runtime and memory are of less concern.

Table 2 shows the results we obtained on the island-style architecture. Column 1 lists the netlist, column 2 lists the size of the smallest square array needed to just fit the netlist, and column 3 lists routing runtimes obtained on using heuristic estimates. Columns 4, 5, and 6 list routing runtimes and compression ratios (shown in brackets) produced by the low-effort associate-with-logic-unit technique, K-Means clustering at empirically determined settings $\left(|\mathrm{S}|=0.06^{*} \mathrm{~N}_{\mathrm{T}}, \mathrm{K}=\mathrm{N}_{\mathrm{L}}\right)$, and K-Means clustering at high-quality settings $\left(|\mathrm{S}|=0.20 * \mathrm{~N}_{\mathrm{T}}, \mathrm{K}=\right.$ $2 * N_{L}$ ) respectively. Routing runtimes are normalized to runtimes produced by heuristic estimates. The compression ratio is defined as the ratio between the size of an exhaustive lookup table and a lookup table that holds cost-to-target estimates for the clusters 
produced by each of the three techniques. The compression ratio is a measure of the memory gap between a version of Pathfinder that uses an exhaustive lookup table and a version that uses costto-target estimates produced by our clustering techniques. Column 7 shows routing runtimes produced by an undirected (no $\mathrm{A}^{*}$ ) search technique.

Across the set of benchmarks, the runtimes produced by our K-Means clustering techniques are approximately $7 \%$ (high-quality settings) and $11 \%$ (empirical settings) slower than the runtimes achieved by heuristically estimating $A^{*}$ costs. Both heuristic and clustering-based estimates are approximately $6 \mathrm{X}$ faster than an undirected searchbased router. Finally, the routing runtimes produced by the associate-with-closest-logic-unit technique is within $5 \%$ of the runtimes produced by either of the K-Means clustering techniques. The near identical runtimes show that the associate-with-closest-logicunit approach presented in Section 3 works as well as a more sophisticated clustering approach on an island-style architecture. The geometric mean of the compression ratios is 30:1 for the associate-withclosest-logic-unit approach and K-Means clustering at empirical settings. The ratio goes down to $18: 1$ for the higher-quality settings. This is to be expected, since we use double the number of starting clusters $\left(\mathrm{K}=2 * \mathrm{~N}_{\mathrm{L}}\right)$ at the higher-quality settings.

\section{Table 2: A comparison of routing runtimes on an island-style architecture.}

\begin{tabular}{|c|c|c|c|c|c|c|}
\hline & \multicolumn{2}{|c|}{$|S|=0.06^{\star} N_{T}$} & \multirow{3}{*}{$\begin{array}{c}\mathrm{S} \mid=0.20^{\star} \mathrm{N}_{\mathrm{T}} \\
\text { K-Means } \\
\left(\mathrm{K}=2^{\star} \mathrm{N}_{\mathrm{L}}\right)\end{array}$} & \multirow[b]{3}{*}{ no $A^{\star}$} \\
\hline & & & \multirow{2}{*}{ Associate } & \multirow{2}{*}{$\begin{array}{c}\text { K-Means } \\
\left(\mathrm{K}=\mathrm{N}_{\mathrm{L}}\right)\end{array}$} & & \\
\hline Netlist & Size & Heur & & & & \\
\hline term1 & $6 \times 6$ & 1.00 & $0.89(17: 1)$ & $1.44(17: 1)$ & $1.22(10: 1)$ & 4.22 \\
\hline $\mathrm{s} 1423$ & $6 \times 6$ & 1.00 & $1.57(20: 1)$ & $1.57(18: 1)$ & $1.14(10: 1)$ & 3.86 \\
\hline i9 & $7 \times 7$ & 1.00 & $1.30(17: 1)$ & $1.30(17: 1)$ & $1.10(10: 1)$ & 3.40 \\
\hline dalu & $8 \times 8$ & 1.00 & $0.93(24: 1)$ & $0.93(22: 1)$ & $1.15(13: 1)$ & 4.04 \\
\hline vda & $9 \times 9$ & 1.00 & $1.20(29: 1)$ & $1.08(32: 1)$ & $1.08(16: 1)$ & 4.78 \\
\hline$x 1$ & $10 \times 10$ & 1.00 & $1.13(20: 1)$ & $0.94(19: 1)$ & $1.17(11: 1)$ & 4.66 \\
\hline rot & $8 \times 8$ & 1.00 & $0.95(26: 1)$ & $1.11(25: 1)$ & $0.89(14: 1)$ & 3.32 \\
\hline pair & $9 \times 9$ & 1.00 & $0.89(30: 1)$ & $0.94(36: 1)$ & $0.94(18: 1)$ & 4.83 \\
\hline apex1 & $11 \times 11$ & 1.00 & $0.97(40: 1)$ & $0.96(37: 1)$ & $1.00(23: 1)$ & 6.03 \\
\hline dsip & $14 \times 14$ & 1.00 & $1.13(22: 1)$ & $1.06(23: 1)$ & $1.07(13: 1)$ & 8.21 \\
\hline ex5p & $12 \times 12$ & 1.00 & $1.03(48: 1)$ & $1.12(48: 1)$ & 1.05 & 7.30 \\
\hline s298 & $16 \times 16$ & 1.00 & $1.58(25: 1)$ & $1.37(23: 1)$ & $1.36(14: 1)$ & 10.38 \\
\hline tseng & $12 \times 12$ & 1.00 & $1.05(27: 1)$ & $1.07(29: 1)$ & $1.04(17: 1)$ & 6.30 \\
\hline alu4 & $14 \times 14$ & 1.00 & $1.09(30: 1)$ & $1.14(30: 1)$ & 1.14 & 7.48 \\
\hline misex3 & $14 \times 14$ & 1.00 & $1.16(40: 1)$ & $1.08(41: 1)$ & $1.05(23: 1)$ & 9.80 \\
\hline apex4 & $13 \times 13$ & 1.00 & $1.10(46: 1)$ & $1.02(45: 1)$ & $1.07(27: 1)$ & 5.04 \\
\hline diffeq & $14 \times 14$ & 1.00 & $1.19(26: 1)$ & $1.13(26: 1)$ & $1.08(15: 1)$ & 5.29 \\
\hline bigkey & $15 \times 15$ & 1.00 & $1.38(26: 1)$ & $1.18(26: 1)$ & $1.08(16: 1)$ & 8.95 \\
\hline seq & $15 \times 15$ & 1.00 & $1.19(37: 1)$ & $1.10(39: 1)$ & $1.05(23: 1)$ & 7.22 \\
\hline des & $15 \times 15$ & 1.00 & $1.20(29: 1)$ & $1.17(29: 1)$ & $1.05(18: 1)$ & 4.35 \\
\hline apex2 & $16 \times 16$ & 1.00 & $1.08(43: 1)$ & $1.09(42: 1)$ & $1.04(26: 1)$ & 8.19 \\
\hline frisc & $22 \times 22$ & 1.00 & $1.08(41: 1)$ & $1.02(41: 1)$ & $1.06(25: 1)$ & 8.56 \\
\hline elliptic & $22 \times 22$ & 1.00 & $1.23(41: 1)$ & $1.00(40: 1)$ & $1.05(24: 1)$ & 10.73 \\
\hline ex1010 & $25 \times 25$ & 1.00 & $0.92(48: 1)$ & $1.15(47: 1)$ & $1.07(29: 1)$ & 9.66 \\
\hline s38584.1 & $29 \times 29$ & 1.00 & $1.07(31: 1)$ & $1.20(31: 1)$ & $1.07(18: 1)$ & 17.07 \\
\hline clma & $33 \times 33$ & 1.00 & $1.03(48: 1)$ & $1.02(48: 1)$ & $1.00(29: 1)$ & 15.25 \\
\hline EOMEAN & & 1.00 & $1.12(30: 1)$ & $1.11(30: 1)$ & $1.07(18: 1)$ & 6.59 \\
\hline
\end{tabular}

Table 3 shows the results that we obtained on HSRA. With the exception of column 2, the settings and columns are identical to Table 2. In this case, column 2 lists the number of logic units in the target device. Across the set of benchmarks, the runtimes produced by our clustering-based techniques are approximately 9\% (higher-quality) and $7 \%$ (empirical settings) faster than the runtimes achieved by heuristically estimating $\mathrm{A}^{*}$ costs. Both heuristic and clustering-based techniques are approximately ten times faster than an undirected search-based router. The runtimes produced by the associate-withclosest-logic-unit technique are approximately $16 \%$ slower than K-Means clustering at empirical settings, and $20 \%$ slower than higher-quality K-Means clustering. This is consistent with our intuition that associating interconnect wires with logic units in a hierarchical structure (Figure 2) will probably produce cost-to-target underestimates.

\section{Table 3: A comparison of routing runtimes on HSRA.}

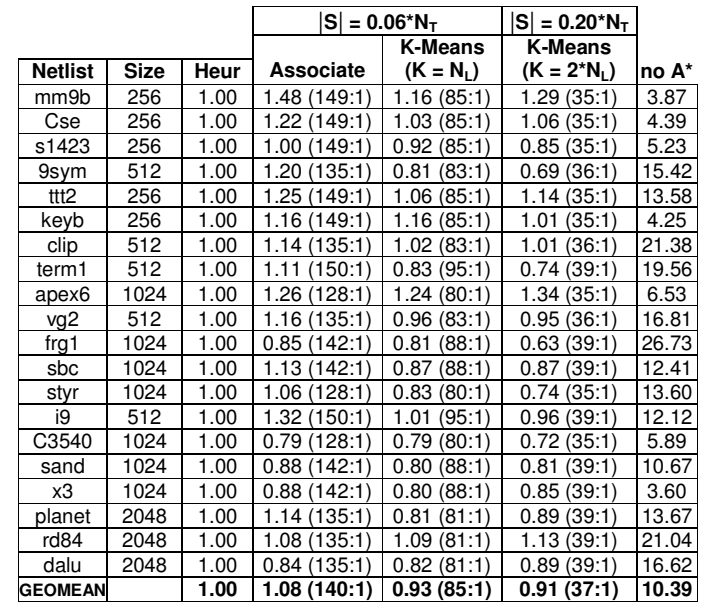

There is a large gap in compression ratio between the associate-with-closest-logic-unit approach and KMeans clustering at empirical settings. In the associate-with-closest-logic-unit approach, each logic unit in the target device is an initial seed. At low subsampling values, a routing wire may be equidistant to several different logic units. Since a routing-wire must eventually be associated with a single logic unit, chances are that a number of logic-units at the end of the clustering process do not have any routing wires associated with them. These logic-unit seeds are eliminated and the number of final clusters is significantly less than the number of starting seeds. This effect is mitigated in K-Means clustering because the initial seeds are a mix of logic units and randomly selected routing wires. Thus, relatively few clusters are eliminated and the compression ratio is lower than the associate-with-logic-unit approach.

\section{Conclusions}

Our goal in this paper was the development of architecture-adaptive $A^{*}$ search techniques that can be used to speed up the Pathfinder algorithm. The 
clustering-based techniques presented in this paper do not rely on architecture-specific heuristics to calculate cost-to-target estimates. This is in direct contrast to previously published techniques $[11,12]$ that explicitly rely on Manhattan distance routability estimates, making them applicable only to islandstyle FPGAs. Our techniques should work on any FPGA that can be represented as a routing graph. The adaptability of our approach is demonstrated in Experiment 3; on an island-style architecture, the runtimes produced by the K-Means clustering approach are within $7 \%$ of heuristic estimates, and $11 \%$ better than heuristic estimates on HSRA. There are several potential benefits of using a clusteringbased approach to calculate cost-to-target estimates:

Memory - During the routing process, the memory requirements of the lookup tables produced by our techniques are $18-30$ times (island-style architecture) and 37 - 140 times (HSRA) less than the exhaustive lookup table proposed by the creators of the Pathfinder algorithm. Thus, our techniques offer adaptability, albeit at significantly smaller memory cost.

Cost of Calculation - The lookup tables produced by our techniques eliminate the task of calculating cost-to-target estimates on the fly during the routing process. Heuristically calculating estimates in the routing inner loop may be expensive when compared to the simple pointer dereferencing operations required to obtain estimates from a lookup table.

Usability Considerations - A production version of a truly architecture-adaptive Pathfinder implementation must be a stand-alone tool that requires minimal user intervention. An architecturespecific cost-to-target estimator may necessitate source code modifications and possible changes to the tool's interface on a per-architecture basis. We feel that users should not be expected to provide any architecture-specific enhancements to speed up Pathfinder. Our techniques do not require any perarchitecture source-code changes, and interface with a routing tool through a cost-to-target lookup table.

Automatically Generated Architectures: During domain-specific reconfigurable architecture generation $[3,4]$, the nature of the reconfigurable device's interconnect structure may be significantly different across application domains. If Pathfinder is used to route netlists on such architectures, then the cost-to-target estimator used by this flow must adapt to different interconnect structures. Expecting the user to modify the flow to produce cost-to-target estimates goes against the underlying philosophy of automatic architecture generation.

\section{Acknowledgements}

This work was supported by grants from the National Science Foundation, Altera Corporation, and the Alfred P Sloan Foundation.

\section{References}

[1] V. Betz, J. Rose and A. Marquardt, Architecture and CAD for Deep-Submicron FPGAs, Kluwer Academic Publishers, Boston MA, 1999.

[2] G. Boriello, C. Ebeling, S Hauck, and S. Burns, "The Triptych FPGA Architecture", IEEE Transactions on VLS Systems Vol. 3 No. 4, IEEE, New York NY, 1995 , pp. $473-482$.

[3] K. Compton, A. Sharma, S. Phillips, and S. Hauck, "Flexible Routing Architecture Generation for Domain-Specific Reconfigurable Subsystems", International Conference on Field Programmable Logic and Applications, Springer-Verlag, New York NY, 2002, pp. $59-68$.

[4] K. Compton, S. Hauck, "Totem: Custom Reconfigurable Array Generation", IEEE Symposium on FPGAs for Custom Computing Machines, IEEE, New York NY, 2001.

[5] T.H. Cormen, C.E. Leiserson, and R.L. Rivest, Introduction to Algorithms, MIT Press, Cambridge MA, 1990.

[6] A. DeHon, "Balancing Interconnect and Computation in a Reconfigurable Computing Array (or, why you don't really want $100 \%$ LUT utilization)", ACM/SIGDA International Symposium on Field-Programmable Gate Arrays, ACM Press, New York NY, 1999, pp. $69-78$.

[7] A. Marquardt, V. Betz and J. Rose, "Speed and Area Tradeoffs in Cluster-Based FPGA Architectures", IEEE Transactions on VLSI Systems Vol. 8 No. 1, IEEE, New York NY, 2000, pp. 84 - 93.

[8] L. McMurchie, and C. Ebeling, "PathFinder: A Negotiation-Based Performance-Driven Router for FPGAs", ACM/SIGDA International Symposium on Field-Programmable Gate Arrays, ACM Press, New York NY, 1995, pp 111-117.

[9] N. Nilsson, Principles of Artificial Intelligence, Morgan Kaufmann, San Francisco CA, 1980.

[10] A Sharma, C. Ebeling, and S. Hauck, "ArchitectureAdaptive Routability-Driven Placement for FPGAs", in International Conference on Field Programmable Logic and Applications, 2005.

[11] J. Swartz, V. Betz and J. Rose, "A Fast RoutabilityDriven Router for FPGAs", ACM/SIGDA International Symposium on Field Programmable Gate Arrays, ACM Press, New York NY, 1998, pp. $140-149$.

[12] R. Tessier, "Negotiated A* Routing for FPGAs", Fifth Canadian Workshop on Field Programmable Devices, 1998. 